Pacific Journal of Mathematics

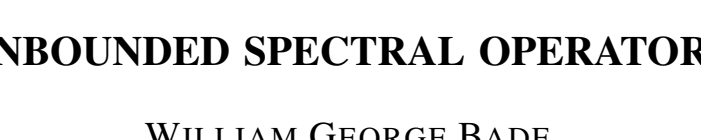




\title{
UNBOUNDED SPECTRAL OPERATORS
}

\author{
WILLIAM G. BADE
}

1. Introduction. Our purpose in the present paper is to study the structure and operational calculus of unbounded spectral operators. Bounded spectral operators have been introduced and studied by N. Dunford in [2] and [3], and the present paper is an investigation in the unbounded case of certain of the results of [3]. Interest in the abstract theory of unbounded spectral operators arises from important results of J. Schwartz [7], who has shown that the members of a large class of differential operators on a finite interval determine unbounded spectral operators in Hilbert space.

Let $B$ denote the Borel subsets of the complex plane, and let $\chi$ be a complex Banach space. We shall call a mapping $E$ from $B$ to projection operators in $\chi$ a resolution of the identity if it is a homomorphism. That is,

$$
\begin{aligned}
& E(e) E(f)=E(e f), \quad E(e) \cup E(f)=E(e \cup f), \quad e, f \in \mathbb{B} \\
& E\left(e^{\prime}\right)=I-E(e), \quad E(\phi)=0, \quad E(p)=I, \quad e \in \mathbb{B} \text {; }
\end{aligned}
$$

$E(e)$ is bounded,

$$
|E(e)| \leq M, \quad e \in \mathbb{B}
$$

and ${ }^{1}$ the vector-valued set function $E(e) x$ is countably additive. Here $\phi$ is the void set, $p$ the plane, and $e^{\prime}$ the complement of $e$ in $p$.

A closed operator $T$ will be called a spectral operator if there is a resolution of the identity $E$ such that:

(1) The domain $D(T)$ of $T$ contains the dense subspace $\chi_{0}=\{x \mid x=E(\sigma) x$, $\sigma \in \mathbb{B}, \sigma$ bounded $\}$.

(2) If $\sigma \in \mathbb{B}, E(\sigma) D(T) \subset D(T)$ and $E(\sigma) T x=T E(\sigma) x, x \in D(T)$.

${ }^{1}$ The last condition is somewhat more restrictive than in [3].

Received March 4, 1953. This paper was prepared under Office of Naval Research contract number onr 609(04). The author is grateful to Professor Dunford for suggesting this investigation.

Pacific J. Math. 4 (1954), $373-392$ 
(3) $\sigma(T, E(\sigma) \mathfrak{X}) \subset \bar{\sigma}$ where $\sigma(T, E(\sigma) \mathcal{X})$ is the spectrum of $T$ in the subspace $E(\sigma) \mathfrak{X}$.

If $\sigma$ is a bounded set, then $T$ is a bounded spectral operator in the subspace $E(\sigma) X$, and in this subspace its structure and operational calculus are known from [3]. The idea of the present paper is to determine the properties of $T$ in $X$ from those of the sequence of approximating bounded spectral operators $T E\left(\sigma_{n}\right)$, where $\left\{\sigma_{n}\right\}$ is an increasing sequence of bounded sets for which

$$
E\left(\begin{array}{ll}
\bigcup_{n=1}^{\infty} & \sigma_{n}
\end{array}\right)=I
$$

We outline briefly the main results:

The simplest type of spectral operator $S$ is that of scalar type:

$$
S x=\lim _{n \rightarrow \infty} \int_{e_{n}} \lambda E(d \lambda) x,
$$

where this limit exists and

$$
e_{n}=\{\lambda|| \lambda \mid \leq n\}
$$

With each spectral operator $T$ we can construct an associated scalar type operator $S$ from its resolution of the identity. One of the principal results of the bounded case is the characterization theorem [3, Theorem 8] that $T$ is a bounded spectral operator if and only if $T=S+N$, where $S$ is a bounded scalar type operator and $N$ is a generalized nilpotent operator commuting with $S$. In the unbounded case the relation of $T$ to $S$ is not so simple, as we shall show by examples. The operator $N=T-S$ (with suitably defined donain) may be bounded but not a generalized nilpotent or even unbounded with spectrum covering the plane. We give a sufficient condition (Theorem 4.1) that $T=S+N$ shall be a spectral operator.

If $S$ is a spectral operator of scalar type, it has an operational calculus exactly analogous to that of an unbounded normal operator in lilbert space (which is an example of a spectral operator). To each Borel measurable function $f$ on $\sigma(S)$ we can assign a densely defined closed operator $f(S)$ which is also a spectral operator of scalar type, the operators corresponding to $f$ and $|f|$ having the same domain. In case $T=S+N$ is a general spectral operator we can, by the formula 


$$
f(T) x=\lim _{p \rightarrow \infty} \sum_{n=0}^{\infty} \frac{N^{n}}{n !} \int_{e_{p}} f^{(n)}(\lambda) E(d \lambda) x,
$$

assign a densely defined operator $f(T)$ to each function analytic and singlevalued in the complement of a set $\theta$ for which $E(\theta)=0$. (Here $\left\{e_{n}\right\}$ is an increasing sequence of compact sets on each of which $f$ is analytic and with $E\left(\cup_{n=1}^{\infty} e_{n}\right)=1$.) However, as we shall show by an example, this operator need not be a spectral operator without other restrictions. If $f$ is a rational function, $f(T)$ is always a spectral operator. Conditions are given to ensure that $f(T)$ is bounded. A result of the calculus is the theorem that a closed operator $T$ with nonempty resolvent set is a spectral operator if and only if $(\lambda I-T)^{-1}$ is a bounded spectral operator for some $\lambda \notin \sigma(T)$. In case $T$ is of the form $T=S+N$, where $N$ is a generalized nilpotent, we obtain quite an extensive operational calculus of spectral operators. In order that $f(T)$ shall be a spectral operator it is sufficient that the singularities of $f(\lambda)$ in the finite plane (with the possible exception of a finite set of poles on $\sigma(T))$ shall not get arbitrarily close to $\sigma(T)$.

2. Closed extensions. In this first section we establish the existence of a closed extension of certain densely defined operators. This result will be the main tool of the paper and it will be convenient to formulate it under rather general conditions. We shall suppose throughout this section the existence of a resolution of the identity $E$.

DEFINITION 2.1. Let $Q$ be an operator defined on a dense subspace $D_{0}(Q)$ of $\mathfrak{X}$. Let there be associated with $Q$ a class $\mathscr{U}$ of Borel sets satisfying:

(a) $\mathfrak{2}$ is closed under finite unions and contains any Borel subset of one of its members;

(b) If $e \in \mathfrak{X}$, then $E(e) \mathfrak{X} \subseteq D_{0}(Q)$ and $Q$ is bounded in $E(e) \mathfrak{X}$;

(c) $E(e) Q E(e)=Q E(e), e \in \mathfrak{U}$;

(d) $\left\{\right.$ contains an increasing sequence $\left\{e_{n}\right\}$ such that $E\left(\cup_{n=1}^{\infty} e_{n}\right)=I$.

Under these conditions we say $Q$ satisfies condition $(\alpha)$ and write

$$
\mathfrak{X}_{\mathfrak{Q}}=\{x \mid x=E(e) x \text { for some } e \in \mathscr{Z}\} \text {. }
$$

An important case occurs when 20 consists of all bounded Borel sets. We shall be interested in finding a particular closed extension of $Q$. The construction will be based on two lemmas. 
L.EMMA 2.1. Let $\left\{d_{n}\right\}$ and $\left\{e_{n}\right\}$ be two increasing sequences of sets from थै $(Q)$ for which

$$
E\left(\bigcup_{n=1}^{\infty} d_{n}\right)=E\left(\bigcup_{n=1}^{\infty} e_{n}\right)=I
$$

If $x \in \mathfrak{X}$, and $\lim _{n \rightarrow \infty}$ ? $E\left(d_{n}\right) x$ exists, then

$$
\lim _{n \rightarrow \infty} Q E\left(e_{n}\right) x=\lim _{n \rightarrow \infty} Q E\left(d_{n}\right) x
$$

Proof. Given $\epsilon>0$, let $m_{0}$ be chosen so that if $m>m_{0}$ then

$$
\left|Q E\left(d_{m}-d_{m_{0}}\right) x\right|<\frac{\epsilon}{3 m^{i}} .
$$

Now, as $E^{\prime}\left(\cup_{m=0}^{\infty} e_{n}\right)=I$ and $Q$ is bounded in $E\left(d_{m_{0}}\right) X$, we can find an $n_{0}$ such that, if $n>n_{0}$,

$$
\left|Q E\left(d_{m_{0}}-e_{n}\right) x\right|<\frac{\epsilon}{3}
$$

For any such fixed $n>n_{0}$ we can, for the same reasons, find an $m_{1}>m_{0}$ so that

$$
\left|Q E\left(e_{n}-d_{m_{1}}\right) x\right|<\frac{\epsilon}{3}
$$

Now, since

$$
E\left(e_{n}\right)-E\left(d_{m_{0}}\right)=E\left(e_{n}-d_{m_{1}}\right)+E\left(e_{n}\right) E\left(d_{m_{1}}-d_{m_{0}}\right)-E\left(d_{m_{0}}-e_{n}\right),
$$

it follows that

$$
\left|Q E\left(e_{n}\right) x-Q E\left(d_{m_{0}}\right) x\right|<\epsilon
$$

DEFINITION 2.2. Let $\left\{e_{n}\right\}$ be any increasing sequence of sets from $\mathcal{A}(Q)$ for which $E\left(\cup_{m=1}^{\infty} e_{n}\right)=I$. We define

$$
D(Q)=\left\{x \mid \lim _{n \rightarrow \infty} Q E\left(e_{n}\right) x \text { exists }\right\}
$$

and set $Q x=\lim _{n \rightarrow \infty} Q E\left(e_{n}\right) x$ for $x \in D(Q)$. 
LEMMA 2.2. The operator? with domain $D(Q)$ is closed and is the minimal closed extension of $Q$ on $\hat{x}_{\text {. }}$. Further, if $x \in D(Q)$, and $e \in \mathcal{R}$, then $E(e) x \in \mathcal{D}(Q)$ and $E(e) Q x=Q E(e) x$. Also, $Q$, with domain $E(e) D(Q)$, is the minimal closed extension in $E(e) X$ of $Q$ on $X_{\mathbb{H}_{1}}, \mathfrak{X}_{1}=\{$ e $\sigma \mid \sigma \in \mathfrak{A}\}$.

Proof. Clearly, first, if $e \in \mathfrak{U}(Q)$ and $x \in D(Q)$, then $Q E(e) x=E(e) Q x$ since we can suppose $e$ a member of the sequence $\left\{e_{n}\right\}$. Now let $x_{n} \in D(Q)$ $(n=1,2, \ldots)$ and

$$
x_{0}=\lim _{n \rightarrow \infty} x_{n}, y_{0}=\lim _{n \rightarrow \infty} Q x_{n} .
$$

For any $m$,

$$
E\left(e_{m}\right) y_{0}=\lim _{n \rightarrow \infty} E\left(e_{m}\right) Q x_{n},
$$

and

$$
\operatorname{QE}\left(e_{m}\right) x_{0}=\lim _{n \rightarrow \infty} Q E\left(e_{m}\right) x_{n}
$$

as $Q$ is bounded in $E\left(e_{m}\right) x$. But since

$$
\left(e_{m}\right) x_{n}=E\left(e_{m}\right) Q x_{n}
$$

we have

$$
\lim _{n \rightarrow \infty} \operatorname{OE}\left(e_{m}\right) x_{0}=\lim _{n \rightarrow \infty} E\left(e_{m}\right) y_{0}=y_{0} .
$$

Thus $x_{0} \in D(Q)$ and $P x_{0}=y_{0}$. Clearly the extension is minimal. Finally let $x \in D(Q), e \in B$. Then

$$
E(e) x=\lim _{n \rightarrow \infty} E\left(e e_{n}\right) x
$$

and

$$
Q E\left(e e_{n}\right)=E(e) Q E\left(e_{n}\right) x
$$

converges to $E(e) Q x$. The last statement follows easily.

We will also need:

LEMMA 2.3. Let $\left\{e_{n}\right\}$ be an increasing sequence of sets from 20 for which 


$$
E\left(\begin{array}{cc}
\bigcup_{n=1}^{\infty} & e_{n}
\end{array}\right)=I .
$$

If, for each $n, \lambda \in \rho\left(Q, E\left(e_{n}\right) \mathfrak{X}\right)$ and

$$
\lim _{n \rightarrow \infty}(\lambda I-Q)^{-1} E\left(e_{n}\right) x
$$

exists for each $x \in \mathfrak{X}$, then $\lambda \in \rho(\eta)$.

Proof. Clearly $\lambda I-Q$ is a closed one-to-one mapping of

$$
D(\lambda I-Q)=D(Q)
$$

into $\mathfrak{X}$. We must show it is onto. Let $x \in \mathfrak{X}$ and

$$
y_{n}=(\lambda I-Q)^{-1} E\left(e_{n}\right) x
$$

Then $\lim _{n \rightarrow \infty} y_{n}=y$ exists by hypothesis, and

$$
\lim _{n \rightarrow \infty}(\lambda I-Q) y_{n}=\lim _{n \rightarrow \infty} E\left(e_{n}\right) x=x .
$$

Hence $y \in D(Q)$ and $(\lambda l-Q) y=x$.

We note that if $T$ is a spectral operator and $T_{0}$ is the closed operator obtained by taking for $\mathcal{A}$ the class of bounded Borel sets and defining $Q x=T x$, $x \in \mathfrak{X}_{2}$, then $T=T_{0}$. Thus a spectral operator has no proper closed extension which is a spectral operator.

3. Scalar type spectral operators. We begin by studying the simplest type of spectral operators, those which can be constructed from a resolution of the identity $E$ by integrating scalar functions. The integral we use for bounded functions over bounded sets is that introduced by Dunford [3, Lemma 6]. We particularly recall the relations

(3.1) $\frac{1}{v(E)} \inf _{\lambda \in e}|f(\lambda)| \leq\left|\int_{e} f(\lambda) E^{\prime}(d \lambda)\right| \leq v(E) \sup _{\lambda \in e}|f(\lambda)|$

and

$$
\int_{e} f(\lambda) g(\lambda) E(d \lambda)=\int_{e} f(\lambda) E(d \lambda) \int_{e} g(\mu) E(d \mu)
$$


where $e$ is a bounded Borel set, $v(E)=4 M$, and $f$ and $g$ are bounded Borel measurable functions. ${ }^{1}$ Ke lenote by $m$ the set of liorel measurable functions $f$ each of which is finite-valued in the complement of a set $\phi_{f}$ for which $E\left(\phi_{f}\right)=0$.

If $f \in M$, we let $थ$ be the class of bouncied Borel sets on which $|f(\lambda)|$ is bounded and take

$$
e_{n}=\{\lambda|| \lambda|\leq n,| f(\lambda) \mid \leq n\} \quad(n=1,2, \cdots) .
$$

We define

$$
f(S) x=\lim _{n \rightarrow \infty} \int_{e_{n}} f(\lambda) E(d \lambda) x
$$

on the set $\nu(f(S))$ of $x$ for which this limit exists. Lemma 2.2 shows that $f(S)$ is a closed operator, and I.emma 2.1 that we would have obtained the same result by using any other increasing sequence $\left\{\sigma_{n}\right\}$ from for which

$$
E\left(\begin{array}{cc}
\infty & \\
U & \sigma_{n}
\end{array}\right)=I
$$

We shall denote by $S$ the operator obtained by taking $f(\lambda)=\lambda$ and call it the scalar operator associated with $E$ (or if $E$ is the resolution of the identity of a spectral operator $T$, we call $S$ the scalar operator associated with $T$ ). Now $S$ is a generalization of an unbounded normal operator in Hilbert space. ${ }^{2}$ The method we have used to construct the operators $f(S)$ is an extension of the method of forming direct sums of iiilbert spaces (see $[6, \mathrm{p} .43]$ ).

THEOREM 3.1. Concerning the operator $f(S)$ wie have:

( I) if $f \in M$, then $L(f(S))=\mathcal{L}(|f|(S))$;

(2) if $f, g \in M$ and $|f(\lambda)| \leq K|g(\lambda)|$, then $U(g(S)) \subset L(f(S))$;

(3) $g(S)$ is bounded if and only if $g$ is essentially bounded with respect to $\{E(e)\}$;

(4) if $f \in M_{\text {and }} g\left(S^{\prime}\right)$ is bounded, then $g(S) D(f(S)) \subset D(f(S))$.

Proof. Wie note that (3) follows from formula (3.1). To prove (1), let

1 The first half of (3.1) does not appear explicitly in [3] but follows from the second half and (3.2).

2 "Maximal normal operator" in the terminology of Stone [8]. 
$\epsilon>0$ be given, and let

$$
\mu=\{\lambda|| f(\lambda) \mid<\epsilon\}
$$

We define $s(\lambda)$ to be $|f(\lambda)|[f(\lambda)]^{-1}$ for $\lambda \notin \mu$, and zero for $\lambda \in \mu$. Then if $x \in \mathcal{D}(f(S))$, for any $n$ we have

$$
\int_{e_{n}}|f(\lambda)| E(d \lambda) x=s(S) \int_{e_{n}-\mu} f(\lambda) E(d \lambda) x+\int_{e_{n} \mu}|f(\lambda)| E(d \lambda) x .
$$

But $|s(S)| \leq v(E)$, and the last term is in norm not greater than $\in v(E)$. It follows that the sequence

$$
\left\{\int_{e_{n}}|f(\lambda)| E(d \lambda) x\right\}
$$

is a Cauchy sequence if

$$
\left\{\int_{e_{n}} f(\lambda) E(d \lambda) x\right\}
$$

also is one. Thus $D(f(S)) \subset D(|f|(S))$. The converse inclusion and (2) are proved similarly. Finally (4) follows from (3.2), since

$$
\int_{e_{n}} f(\lambda) E(d \lambda) g(S) x=\int_{e_{n}} f(\lambda) g(\lambda) E(d \lambda) x=g(S) \int_{e_{n}} f(\lambda) E(d \lambda) x
$$

THEовем 3.2. Let $f$ and $g \in M$.

(1) If $x \in D(f(S)) \cap D(g(S))$, then $x \in D((f+g)(S))$ and $[f(S)+$ $g(S)] x=(f+g)(S) x$.

(2) If $x \in D(g(S))$ and $g(S) x \in D(f(S))$, then $x \in D((f g)(S))$ and $f(S) g(S) x=(f g)(S) x$.

Proof. (1) is clear. For (2), let 21 consist of the bounded Borel sets on which both $f(\lambda)$ and $g(\lambda)$ are bounded, and let

$$
e_{n}=\{\lambda|| f(\lambda)|,| g(\lambda) \mid \text { and }|\lambda| \leq n\}
$$

Then, for any $n$, 


$$
\begin{aligned}
\int_{e_{n}} f(\lambda) E(d \lambda) g(S) x & =\lim _{m \rightarrow \infty} \int_{e_{n}} f(\lambda) E(d \lambda) \int_{e_{m}} g(\mu) E(d \mu) \\
& =\int_{e_{n}} f(\lambda) g(\lambda) E(d \lambda) x,
\end{aligned}
$$

since $\int_{e_{n}} f(\lambda) E(d \lambda)$ is a bounded operator. Thus $f(S) g(S) x=(f g)(S) x$.

For the next theorem we will need a lemma which it will be convenient later to have formulated for a general spectral operator.

LEMMA 3.1. If $T$ is a spectral operator $E(\sigma(T))=I_{s}$ and if $\left\{e_{n}\right\}$ is an increasing sequence of bounded Borel sets for which

$$
E\left(\bigcup_{n=1}^{\infty} e_{n}\right)=I,
$$

then

$$
\sigma(T)=\overline{\bigcup_{n=1}^{\infty} \sigma\left(T, E\left(e_{n}\right) X\right)} .
$$

Proof. The argument follows that of $[3$, Theorem 1]. Let

$$
\mu=\overline{\bigcup_{n=1}^{\infty} \sigma\left(T, E\left(e_{n}\right) X\right)} .
$$

Clearly $\mu \subset \sigma(T)$. If $\sigma$ is a closed subset of $\mu^{\prime}$, then, for each $n, \sigma\left(T, E\left(\sigma e_{n}\right) X\right)$ is a subset of both $\sigma$ and $\sigma\left(T, E\left(e_{n}\right) \mathfrak{X}\right)$. Thus

$$
E\left(\sigma e_{n}\right)=0, \quad E(\sigma)=0, \quad \text { and } \quad E\left(\mu^{\prime}\right)=0 .
$$

Hence $E(\mu)=I$ and $\mu=\sigma(T)$.

THEOREM 3.3. If $f \in M$, then $f(S)$ is a spectral operator whose resolution of the identity is given by

$$
E_{f}(e)=E\left(f^{-1}(e)\right)
$$

and spectrum by 


$$
\sigma(f(S))=\bigcap_{E(e)=I} \overline{f(e)} .
$$

Proof. I et $\sigma$ be a fixed Borel set. If $\lambda_{0} \not \bar{\sigma}$ then

$$
g(\lambda)=\left(\lambda_{0}-f(\lambda)\right)^{-1} / f^{-1}(\sigma)
$$

is bounded, and the equations

$$
\begin{aligned}
& g(S)\left(\lambda_{0} I-f(S)\right) x=x, \quad x \in E(\sigma) L(f(S)), \\
& \left(\lambda_{0} I-f(S)\right) g(S) x=x, \quad x \in E(\sigma) \mathcal{X},
\end{aligned}
$$

show $\lambda_{0} I-f(S)$ is a closed one-to-one map of $E(\sigma) D(f(S))$ onto $E(\sigma) x$. Thus $\sigma\left(f(S), E_{f}(\sigma) \mathcal{X}\right) \subset \bar{\sigma}$.

Now let

$$
e_{n}=\{\lambda|| \lambda|\leq n,| f(\lambda) \mid \leq n\}
$$

By $\mid 3$, Theorem $16 \mid$,

$$
o\left(f(S), E\left(e_{n}\right) X\right)=\bigcap_{E(e)=E\left(e_{n}\right)} \overline{f(e)}=\mu_{n}
$$

Now, by I.emma 3.1,

$$
\sigma(f(S))=\overline{\bigcup_{n=1}^{\infty} \mu_{n}} .
$$

Jet

$$
\mu=\bigcap_{E(e)=I} \overline{f(e)} .
$$

Clearly $\mu_{n} \subset \mu$ for each $n$. If

$$
\lambda \notin \bigcup_{n=1} \mu_{n},
$$

we can pick a $\delta>0$ and for each $n$ a ljorel set $\sigma_{n} \subset e_{n}$ such that

$$
E\left(\sigma_{n}\right)=E\left(e_{n}\right) \text { and dist. }\left(\lambda, \overline{f\left(\sigma_{n}\right)}\right)>\delta \text {. }
$$


Now if

$$
\sigma_{0}=\bigcup_{n=1}^{\infty} \sigma_{n},
$$

then $E\left(\sigma_{0}\right)=I$ and $\lambda \notin \overline{f\left(\sigma_{0}\right)}$, and thus $\lambda \notin \mu$. Hence

$$
\mu=\overline{\bigcup_{n=1}^{\infty} \mu_{n}}=\sigma(f(S))
$$

4. The relation of $T$ to its scalar operator. One of Dunford's principal results for bounded spectral operators is the characterization theorem [3, Theorem $8]$ that $T$ is a bounded spectral operator if and only if $T=S+N$, where

$$
S=\int \lambda E(d \lambda)
$$

is the associated scalar type operator and $N$ is a generalized nilpotent operator commuting with $T$. The absence of such a theorem in the unbounded case greatly complicates the theory. While in each subspace $E(\sigma) \mathfrak{X}, \sigma$ bounded, $N=T-S$ will be a generalized nilpotent, the natural closed extension provided by Lemma 2.2 of $N$ on $\mathcal{X}_{2 Y}$, ( 2\} the class of bounded Borel sets) may be bounded but not a generalized nilpotent, or even unbounded. We now construct two examples which exhibit these possibilities.

EXAMPLE 1. For each $n$, let $\aleph_{2}$ be $n$-dimensional unitary space and let $\mathscr{S}_{2}$ be the space of sequences $\left\{x_{n}\right\}$, where

$$
\begin{gathered}
x_{n}=\left(\xi_{1 n}, \xi_{2 n}, \cdots, \xi_{n n}\right) \in \xi_{\xi_{n}} \\
\left|x_{n}\right|=\left(\sum_{i=1}^{n}\left|\xi_{i n}\right|^{2}\right)^{1 / 2}, \quad|x|=\left(\sum_{n=1}^{\infty}\left|x_{n}\right|^{2}\right)^{1 / 2} .
\end{gathered}
$$

Then $\sqrt{2}$ is a Hilbert space. We denote by $E(n)$ the orthogonal projection mapping $\mathscr{S}_{2}$ onto $\mathscr{S}_{n}$. The Boolean algebra $E$ of projections

$$
E(\sigma)=\sum_{n \in \sigma} E(n),
$$

where $\sigma$ is any subset of the positive integers, is a resolution of the identity 
of the self-adjoint operator $S$ which we define in $\mathscr{F}_{n}$ by

$$
S x_{n}=\left(n \xi_{1 n}, \cdots, n \xi_{n n}\right)
$$

and extend by Lemma 2.2 to

$$
D(S)=\left\{\left.x\left|\sum_{n=1}^{\infty}\right| S x_{n}\right|^{2}<\infty\right\} .
$$

The operator $N$ we define in $\mathscr{K}_{n}$ by

$$
N x_{n}=\left(0, n \xi_{1 n}, n \xi_{2 n}, \cdots, n \xi_{n-1 n}\right) \text {. }
$$

The extension to $\sqrt{2}$ yields an operator of norm one which is nilpotent of order $n$ on $\mathscr{F}_{n}$. Whe shall show that the operator

$$
T=S+N,
$$

is a spectral operator. Let $\sigma$ be any subset of the positive integers and $\alpha \notin \sigma$. If $n \in \sigma$, the operator

$$
R_{\alpha}\left(T, \mathcal{F}_{n}\right)=\sum_{i=0}^{n-1} \frac{N^{i} E(n)}{(\alpha-n)^{i+1}}
$$

is the resolvent operator of $T$ in the subspace $\widetilde{2}_{n}$. Because of the quadratic nature of the norm in Hilbert space, $a$ will be in the resolvent set of $T$ in $E(\sigma) \mathscr{F}_{2}$ if and only if $\left|R_{\alpha}\left(T, \mathscr{F}_{n}\right)\right|$ is uniformly bounded for all $n$ in $\sigma$. But this is satisfied; in fact,

$$
\lim _{n \rightarrow \infty}\left|R_{\alpha}\left(T, \mathscr{S}_{n}\right)\right|=0
$$

where $n$ is not restricted to $\sigma$. For, given $1>\epsilon>0$, we can pick an $n_{0}$ so large that

$$
|\alpha-n|^{-1}<\frac{\epsilon}{2}
$$

Then, if $n>n_{0}$,

$$
\left|R_{\alpha}\left(T, \mathscr{S}_{n}\right)\right| \leq \sum_{i=0}^{n-1} \frac{1}{|\alpha-n|^{i+1}}<\epsilon .
$$


Thus $\sigma\left(T, E(\sigma) \mathscr{F}_{2}\right) \subset \sigma$, and $T$ is a spectral operator. To show that $N$ is not a generalized nilpotent, let $x=\left\{x_{i}\right\}$, where

$$
x_{i}=\left(2^{-i / 2}, 0,0, \cdots, 0\right) \text {. }
$$

Then $|x|=1$, but

$$
\left|N^{n} x\right|^{1 / n}=\frac{1}{\sqrt{2}}
$$

The transformation $N$ is of a type studied by H. Hamburger [4].

EXAMPLE 2. In this case let $\tilde{S}_{n}$ be two-dimensional unitary space for each $n$, and form $\mathscr{F}_{2}$ as the Hilbert space of sequences $\left\{x_{n}\right\}$ with $x_{n}=\left(\xi_{1 n}, \xi_{2 n}\right) \in$ $\mathfrak{F}_{n}$ as before. In $\mathscr{F}_{n}$ we define

$$
\begin{aligned}
& S x_{n}=\left(n \xi_{1 n}, n \xi_{2 n}\right), \\
& N x_{n}=\left(0, n \xi_{1 n}\right),
\end{aligned}
$$

and $T=S+N$. Then

$$
D(T)=\left\{\left.x\left|\sum_{n=1}^{\infty}\right| T x_{n}\right|^{2}<\infty\right\}
$$

with similar expressions for $D(S)$ and $D(N)$. As $D(S) \subset D(N)$, we have $D(T)=D(S)$. Now $N$ has the entire plane as its spectrum since, clearly, $0 \in \sigma(N)$, and, if $\beta \neq 0$, the formula

$$
R_{\beta}\left(N, \mathcal{L}_{n}\right) x_{n}=\left(\frac{\xi_{1 n}}{\beta^{2}}, \frac{n \xi_{1 n}}{\beta^{2}}+\frac{\xi_{2 n}}{\beta}\right)
$$

shows that $\left|R_{\beta}\left(N, \mathscr{F}_{n}\right)\right|$ is unbounded with $n$. However, $T$ is a spectral operator. If $\sigma$ is a set of integers and $\alpha \notin \sigma$ then, for $n \in \sigma$,

$$
R_{\alpha}\left(T, \mathcal{\zeta}_{n}\right) x_{n}=\left(\frac{\xi_{1 n}}{\alpha-n}, \frac{n \xi_{1 n}}{(\alpha-n)^{2}}+\frac{\xi_{2 n}}{(\alpha-n)}\right)
$$

Thus $\left|R_{\alpha}\left(T, \mathscr{F}_{n}\right)\right|$ is bounded, $n \in \sigma$. 
The last example shows the degree of pathology that may arise. It is interesting that we do have the following result which covers the case of Example 1.

THEOREM 4.1. Let $S$ be an unbounded scalar type operator, and let $N$ be a bounded operator which commutes with the resolution of the identity for $S$ and is a generalized nilpotent on each of the subspaces $E(\sigma) \mathfrak{X}, \sigma$ bounded. Then $T=S+N$ is a spectral operator with the same resolution of the identity.

Proof. The relation $\sigma(T, E(\sigma) \mathfrak{X}) \subset \bar{\sigma}$ is clearly satisfied for all bounded Borel sets. Let $\sigma$ be an unbounded Borel set and let

$$
e_{n}=\{\lambda|| \lambda \mid \leq n\}
$$

By [3, Lemma 3], the resolvent of $T$ in $E\left(\sigma e_{n}\right) \mathfrak{X}$ is given by

$$
(\lambda I-T)^{-1}=\sum_{i=0}^{\infty} N^{i} \int_{\sigma e_{n}} \frac{E(d \mu)}{(\lambda-\mu)^{i+1}} .
$$

We conclude the proof by showing that

$$
\lim _{n \rightarrow \infty}(\lambda I-T)^{-1} E\left(\sigma e_{n}\right) x
$$

exists for each $x \in E(\sigma) \nVdash$ and applying Lemma 2.3 in that subspace. We show in fact that the series

$$
\sum_{i=0}^{\infty} N^{i} \int_{\sigma} \frac{E(d \mu)}{(\lambda-\mu)^{i+1}}
$$

converges. For given $0<\epsilon<1$, we may pick $n_{0}$ so large that

$$
2|N|<\epsilon \operatorname{dist}\left(\lambda, e_{n_{0}}^{\prime}\right), \quad 2 v(E)<\operatorname{dist}\left(\lambda, e_{n_{0}}^{\prime}\right),
$$

and pick an $n_{1}>n_{0}$ such that for any $m$ and $n$ with $m>n>n_{1}$,

$$
\left|\sum_{i=n}^{m} N^{i} \int_{\sigma e_{n_{0}}} \frac{E(d \mu)}{(\lambda-\mu)^{i+1}}\right|<\frac{\epsilon}{2} .
$$

Then, using (3.1), we get 


$$
\begin{aligned}
\left|\sum_{i=n}^{m} N^{i} \int_{\sigma} \frac{E(d \mu)}{(\lambda-\mu)^{i+1}}\right| & <\frac{\epsilon}{2}+\sum_{i=n}^{m}|N|^{i}\left|\int_{\sigma e_{n_{0}}^{\prime}} \frac{E(d \mu)}{(\lambda-\mu)^{i+1}}\right| \\
& <\frac{\epsilon}{2}+\frac{v(E)}{\operatorname{dist}\left(\lambda, e_{n_{0}}^{\prime}\right)} \sum_{i=n}^{m} \frac{\epsilon^{i}}{2^{i}}<\epsilon .
\end{aligned}
$$

5. Operational calculus for a general spectral operator. When $T$ is a bounded operator and $f$ is a function analytic on $\sigma(T)$, it is well known $[1 ; 9]$ that a comprehensive operational calculus is obtained by defining

$$
f(T)=\frac{1}{2 \pi i} \int_{C} f(\lambda)(\lambda I-T)^{-1} d \lambda
$$

where $C$ is a bounded positively oriented contour containing $\sigma(T)$ and excluding the singularities of $f$. Also,

$$
\sigma(f(T))=f(\sigma(T)) .
$$

Moreover, in the case that $T(=S+N)$ is a bounded spectral operator, Dunford has shown [3, Theorem 9] that the operator $f(T)$ may be expressed in terms of the values of $f$ and its derivatives on $\sigma(T)$ by the formula

$$
f(T)=\sum_{n=0}^{\infty} \frac{N^{n}}{n !} \int_{\sigma(T)} f^{(n)}(\lambda) E(d \lambda),
$$

the series converging absolutely in the uniform operator topology. We shall make formula (5.3) the basis of an operational calculus in the unbounded case.

Given an unbounded spectral operator $T$, we denote by $R$ the class of functions $f$ each analytic and single-valued in the complement of a closed set $\theta_{f}$ for which $E\left(\theta_{f}\right)=0$. If for $f \in R$ we take

$$
e_{n}=\left\{\lambda|| \lambda \mid \leq n, \operatorname{dist}\left(\lambda, \theta_{f}\right) \geq \frac{1}{n}\right\},
$$

then $\left\{e_{n}\right\}$ is an increasing sequence of closed sets for which

$$
E\left(\bigcup_{n=1}^{\infty} e_{n}\right)=I,
$$


and on each of which $f$ is analytic. Moreover, $T=S+N$ is a bounded spectral operator in $E\left(e_{n}\right) X$. Defining

$$
f(T) x=\lim _{n \rightarrow \infty} \sum_{i=0}^{\infty} \frac{N^{i}}{i !} \int_{e_{n}} f^{(i)}(\lambda) E(d \lambda) x
$$

on the set $D(f(T))$ of $x$ for which this limit exists, we obtain via Lemma 2.2 a closed densely defined operator. The class $R$ is closed under sums and products, and by an argument exactly analogous to that of Theorem 3.2 we obtain:

THEOREM 5.1. Let $f$ and $g \in R$.

(1) If $x \in D(f(T)) \cap D(g(T))$, then $x \in D((f+g)(T))$ and $(f(T)+$ $g(T)) x=(f+g)(T) x$.

(2) If $x \in D(g(T))$ and $g(T) x \in D(f(T))$, then $x \in D((f g)(T))$ and $f(T) g(T) x=(f g)(T) x$.

As we show now by an example, the operator $f(T)$ need not be a spectral operator. Let $T$ be the operator of Example 2 whose spectrum is the set of positive integers. Taking

$$
f(\lambda)=\sqrt{2} \operatorname{cosec} \pi\left(\lambda+\frac{1}{4}\right)
$$

we see that the spectrum of $f(T)$ in $E(\sigma) \mathcal{S}_{2}$ for $\sigma$ any finite subset of $\sigma(T)$ is the range of $f(\lambda)$ on $\sigma$, that is, lies in the pair of points \pm . By Lemma 3.1, this must be true also of the closed operator $f(T)$ on $D(f(T))$ if it is a spectral operator. However, $0 \in \sigma(f(T))$ since, for $x_{n} \in \mathscr{F}_{n}$,

$$
[f(T)]^{-1} x_{n}=\frac{(-1)^{n}}{2}\left(\xi_{1 n}, n \pi \xi_{1 n}+\xi_{2 n}\right),
$$

showing that the norm of $[f(T)]^{-1}$ in $\mathscr{L}_{n}$ is unbounded with $n$. In fact, $\sigma(f(T))$ is the whole plane.

In connection with Example 1, it is worth noting that there are bounded operators which are spectral operators on each of an increasing sequence $E\left(e_{n}\right) \mathfrak{X}$ of subspaces for which

$$
E\left(\bigcup_{n=1}^{\infty} e_{n}\right)=I
$$


without being spectral operators on $\chi$. Such an operator in the case of Example 1 is given by $S^{-1}+N$, where

$$
e_{n}=\{p \mid 1 \leq p \leq n\}
$$

We now give conditions under which $f(T)$ is a spectral operator.

THEOREM 5.2. Let $T$ be a spectral operator, and let $f$ be analytic on $\sigma(T)$ with the exception of a finite set $\theta=\left(p_{1}, p_{2}, \cdots, p_{k}\right)$ of poles for which $E(\theta)=0$, and let $f$ be either analytic at infinity or have a pole there. Then $f(T)$ is a spectral operator with resolution of the identity

$$
E_{f}(e)=E\left(f^{-1}(e)\right)
$$

and spectrum

$$
\sigma(f(T))=\overline{f(\sigma(T))} \text {. }
$$

For the proof we shall need the following lemma:

LEMMA 5.1. Let $f$ and $T$ satisfy the conditions of Theorem 5.2. Then $\sigma(f(T)) \subset \overline{f(\sigma(T))}$.

Proof. Clearly we can suppose that $\overline{f(\sigma(T))}$ is not the entire plane. Let $\lambda_{0} \notin \overline{f(\sigma(T))}$, and define the function $g(\lambda)$ to be $\left[\lambda_{0}-f(\lambda)\right]^{-1}$ where $f$ is analytic and zero at the poles of $f$. Then $g$ is analytic on $\sigma(T)$ and at infinity. To show that $g(T)$ is a bounded operator, we can suppose that $\sigma(T)$ is not the whole plane, since otherwise $g$ is constant. Now A.E. Taylor [10] has shown that if $T$ is a closed operator whose spectrum does not cover the plane, and $g$ is a function analytic on $\sigma(T)$ and at infinity, then there is an unbounded Cauchy domain $D$ such that $\sigma(T) \subset D, \bar{D}$ is contained in the domain of $g$, and an operational calculus is established by defining

$$
g[T]=g(\propto) I+\int_{K} g(\lambda)(\lambda I-T)^{-1} d \lambda,
$$

where $K$ is the positively oriented bounded contour forming the boundary of $D$. The operator $g[T]$ is bounded, and, in the case $T$ is bounded, $g[T]=g(T)$, the operator of (5.1). Now, recalling the equivalence of (5.1) and (5.3) when $T$ is a bounded spectral operator, we let

$$
e_{n}=\sigma(T) \cap\left\{\lambda|| \lambda \mid \leq n_{,} \text {dist }(\lambda, \theta) \geq \frac{1}{n}\right\},
$$


and note that

$$
g[T]=\sum_{i=0}^{\infty} \frac{N^{i}}{i !} \int_{e_{n}} g^{(i)}(\lambda) E(d \lambda)
$$

in $E\left(e_{n}\right) \nVdash$. Thus, in $\nVdash$,

$$
g[T]=\lim _{n \rightarrow \infty} \sum_{i=0}^{\infty} \frac{N^{i}}{i !} \int_{e_{n}} g^{(i)}(\lambda) E(d \lambda)=g(T) .
$$

Moreover, $g(T)=\left[\lambda_{0} I-f(T)\right]^{-1}$ in $E\left(e_{n}\right) \mathfrak{X}$. Thus, by Lemma 2.3, $\lambda_{0} \notin \sigma(f(T))$.

Proof of Theorem 5.2. Let $\sigma$ be a fixed Borel set. Then

$$
\sigma\left(T, E\left(f^{-1}(\sigma)\right) X\right) \subset \overline{f^{-1}(\sigma)} .
$$

We now apply either $(5.2)$ or the preceding lemma in the subspace $E\left(f^{-1}(\sigma)\right) \nVdash$, depending on whether or not $f^{-1}(\sigma)$ is a bounded set, to conclude that

$$
\sigma\left(f(T), E\left(f^{-1}(\sigma)\right) \mathfrak{X}\right) \subset f \overline{\left(f^{-1}(\sigma)\right)} \subset \bar{\sigma} .
$$

That $\sigma(f(T))=\overline{f(\sigma(T))}$ follows from (5.2) and Lemma 3.1.

CoR OLLARY. Any polynomial in a spectral operator is a spectral operator. A closed operator $T$ is a spectral operator if and only if, for some $\lambda_{0} \notin \sigma(T)$, $\left(\lambda_{0} I-T\right)^{-1}$ is a bounded spectral operator.

Proof. The first statement is clear, as is the necessity of the second. For the sufficiency we note that

$$
T=f\left(\left(\lambda_{0} I-T\right)^{-1}\right), \quad \text { where } f(\lambda)=\lambda_{0}-\frac{1}{\lambda}
$$

If we restrict $N$ to be a generalized nilpotent we obtain a broad operational calculus of spectral operators. All we need require of an analytic function $f$ is that its singularities in the finite plane (with the exception of a finite set of poles as before) shall not be arbitrarily close to $\sigma(T)$.

THEOREM 5.3. Let $T$ be a spectral operator and $T=S+N$, where $N$ is a generalized nilpotent. Let $f$ be a function for which there exists a constant $r>0$ such that $f$ is analytic (with the possible exception of a finite set 
$\theta=\left(p_{1}, \cdots, p_{k}\right)$ of poles for which $\left.E(\theta)=0\right)$ in the open set

$$
\mu_{f}=\{\lambda \mid \operatorname{dist}(\lambda, \sigma(T))<r\} .
$$

Then $f(T)$ is a spectral operator whose resolution of the identity and spectrum are given by (5.3) and (5.4). The class of such functions is closed under sums and products. If $f$ if bounded on $\mu_{f}$, then $f(T)$ is bounded.

The proof proceeds exactly as before once we have:

Lemma 5.2. If $f$ satisfies the conditions of Theorem 5.3, then

$$
\sigma(f(T)) \subset \overline{f(\sigma(T))} .
$$

Proof. Let $f$ and $r$ be given and $\lambda_{0} \notin \overline{f(\sigma(T))}$. Again we define $g(\lambda)$ to be $\left(\lambda_{0}-f(\lambda)\right)^{-1}$ where $f$ is analytic and zero at the poles of $f$. Then as $\lambda_{0} \notin \overline{f(\sigma(T))}$ there is a constant $s>0$ such that $g$ is analytic and bounded in

$$
\mu_{g}=\{\lambda \mid \operatorname{dist}(\lambda, \sigma(T))<2 s\} .
$$

The formula

$$
g^{(n)}(\lambda)=\frac{n !}{2 \pi i} \int_{C} \frac{g(\mu)}{(\mu-\lambda)^{n+1}} d \mu,
$$

where $C$ is a circle of radius $s$, shows that if $|g(\lambda)|<K$ on $\mu_{g}$, then

$$
\left|\frac{g^{(n)}(\lambda)}{n !}\right|<K s^{-n},
$$

Since

$$
\lim _{n \rightarrow \infty}\left|N^{n}\right|^{1 / n}=0
$$

the series

$$
g(T)=\sum_{n=0}^{\infty} \frac{N^{n}}{n !} \int_{\sigma(T)} g^{(n)}(\lambda) E(d \lambda)
$$

converges in the uniform operator topology. Moreover, if

$$
e_{n}=\sigma(T) \cap\left\{\lambda|| \lambda \mid \leq n, \text { dist }(\lambda, \theta) \geq \frac{1}{n}\right\},
$$


$g(T)$ is the resolvent of $f(T)$ on $E\left(e_{n}\right) \hat{X}$. Application of Lemma 2.3 shows that $\lambda_{0} \notin \sigma(f(T))$.

\section{REFERENCES}

1. N. Dunford, Spectral theory 1. Convergence to projections, Trans. Amer. Math. Soc. 54 (1943), $185-217$.

2. - Spectral theory II. Resolutions of the identity, Pacific J. Math. 2 (1952), 559-614.

3. , Spectral operators, Pacific J. Math. 4 (1954), $321-354$.

4. H. Hamburger, Five notes on a generalization of quasi-nilpotent transformations in Hilbert space, Proc. London Math. Soc., 3rd series, 1 (1951), 494-512.

5. E. Hille, Functional analysis and semi-groups, Amer. Math. Soc. Coll. Publications, Vol. 31, New York, 1948.

6. B. Nagy, Spektraldarstellung linearer Transformationen des Hilbertschen Raumes, Berlin, 1942.

7. J. Schwartz, Perturbations of spectral operators and applications, Pacific J. Math. 4 (1954), $415-458$.

8. M.H. Stone, Linear transformations in Hilbert space and their applications to analysis, Amer. Math. Soc. Coll. Publications, Vol. 15, New York, 1932.

9. A. E. Taylor, Analysis in complex Banach spaces, Bull. Amer. Math. Soc. 49 (1943), $652-669$.

10. $189-224$.

Spectral theory of closed distributive operators, Acta Math. 84 (1950),

\section{YALE UNIVERSITY}




\section{PACIFIC JOURNAL OF MATHEMATICS}

\section{EDITORS}

\author{
M.M. SCHIFFE R* \\ Stanford University \\ Stanford, California \\ E. HEWITT \\ University of Washington \\ Seattle 5, Washington
}

R.P. DILWORTH

California Institute of Technology Pasadena 4, California

E.F. BECKENBACH**

University of California

Los Angeles 24, California

\section{ASSOCIATE EDITORS}

$\begin{array}{llll}\text { H. BUSEMANN } & \text { P.R. HALMOS } & \text { BORGE JESSEN } & \text { J. J. STOKER } \\ \text { HERBERT FEDERER } & \text { HEINZ HOPF } & \text { PAUL LÉVY } & \text { E.G. STRAUS } \\ \text { MARSHALL HALL } & \text { R.D. JAMES } & \text { GEORGE PÓLYA } & \text { KÔSAKU YOSIDA }\end{array}$

\section{SPONSORS}

UNIVERSITY OF BRITISH COLUMBIA CALIFORNIA INSTITUTE OF TECHNOLOGY UNIVERSITY OF CALIFORNIA, BERKELEY UNIVERSITY OF CALIFORNIA, DAVIS UNIVERSITY OF CALIFORNIA, LOS ANGELES UNIVERSITY OF CALIFORNIA, SANTA BARBARA UNIVERSITY OF NEVADA OREGON STATE COLLEGE UNIVERSITY OF OREGON
UNIVERSITY OF SOUTHERN CALIFORNIA STANFORD RESEARCH INSTITUTE STANFORD UNIVERSITY WASHINGTON STATE COLLEGE UNIVERSITY OF WASHINGTON

AMERICAN MATHEMATICAL SOCIETY HUGHES AIRCRAFT COMPANY

Mathematical papers intended for publication in the Pacific Journal of Mathematics should be typewritten (double spaced), and the author should keep a complete copy. Manuscripts may be sent to any of the editors. Manuscripts intended for the outgoing editors should be sent to their successors. All other communications to the editors should be addressed to the managing editor, E.G. Straus, at the University of California Los Angeles 24, California.

50 reprints of each article are furnished free of charge; additional copies may be obtained at cost in multiples of 50 .

The Pacific Journal of Mathematics is published quarterly, in March, June, September, and December. The price per volume (4 numbers) is $\$ 12.00$; single issues, $\$ 3.50$; back numbers (Volumes $1,2,3$ ) are available at $\$ 2.50$ per copy. Special price to individual faculty members of supporting institutions and to individual members of the American Mathematical Society: $\$ 4.00$ per volume; single issues, $\$ 1.25$.

Subscriptions, orders for back numbers, and changes of address should be sent to the publishers, University of California Press, Berkeley 4, California.

Printed at Ann Arbor, Michigan. Entered as second class matter at the Post Office, Berkeley, California.

* To be succeeded in 1955, by H.L. Royden, Stanford University, Stanford, California.

** To be succeeded in 1955, by E.G. Straus, University of California, Los Angeles 24, Calif.

UNIVERSITY OF CALIFORNIA PRESS - BERKELEY AND LOS ANGELES 


\section{Pacific Journal of Mathematics \\ Vol. 4, No. $3 \quad$ July, 1954}

Nelson Dunford, Spectral operators ........................ 321

John Wermer, Commuting spectral measures on Hilbert space.......... 355

Shizuo Kakutani, An example concerning uniform boundedness of spectral measures...................................... 363

William George Bade, Unbounded spectral operators .............. 373

William George Bade, Weak and strong limits of spectral operators ...... 393

Jacob T. Schwartz, Perturbations of spectral operators, and applications. I. Bounded perturbations ........................... 415

Mischa Cotlar, On a theorem of Beurling and Kaplansky............... 459

George E. Forsythe, Asymptotic lower bounds for the frequencies of certain polygonal membranes ............................... 467 